\title{
Large Deletion of the Peroxisomal Acyl-CoA Oxidase Gene in Pseudoneonatal Adrenoleukodystrophy
} \author{
Beatrice Foumier, ${ }^{\star \ddagger}$ Jean-Marie Saudubray, ${ }^{\ddagger}$ Bernard Benichou, ${ }^{\$}$ Stanislas Lyonnet, ${ }^{\ddagger}$ Amold Munnich, ${ }^{\ddagger}$ Hans Clevers,
and Bwee Tien Poll-The \\ *University Children's Hospital Wilhelmina Kinderziekenhuis and DNA Laboratory University Hospital Utrecht, 3512 LK Utrecht, The \\ Netherlands; ${ }^{\ddagger}$ Unité de Recherches sur les Handicaps Génétiques de l'Enfant, Institut National de la Santé et de la Recherche Médicale \\ (INSERM) U-12 et Département de Pédiatrie, Hôpital des Enfants-Malades, 75743 Paris, France; ${ }^{\S}$ Centre National de la Recherche \\ Scientifique UPR420, Villejuif cedex, France; and "Department of Immunology, University Hospital Utrecht, 3508 GA Utrecht, \\ The Netherlands
}

\begin{abstract}
We have cloned the cDNA encoding human peroxisomal acyl-CoA oxidase, the first enzyme in the peroxisomal $\beta$ oxidation of very long chain fatty acids. Its nucleotide sequence was found to be highly homologous $(85 \%)$ to the rat cDNA counterpart.

An 88\% homology between rat and human was found in the COOH-terminal end of the CDNA which includes the Ser-Lys-Leu peroxisomal targeting signal common to many peroxisomal proteins. The gene spans $\sim 30-40 \mathrm{~kb}$ and is poorly polymorphic. Southern blot analyses were performed in two previously reported siblings with an isolated peroxisomal acyl-CoA oxidase deficiency (pseudoneonatal adrenoleukodystrophy). A deletion of at least $17 \mathrm{~kb}$, starting downstream from exon 2 and extending beyond the $3^{\prime}$ end of the gene, was observed in the two patients. These observations provide a molecular basis for the observed acyl-CoA oxidase deficiency in our family. In addition, our study will enable the characterization of the genetic defect in unrelated families with suspected acyl-CoA oxidase disorders. (J. Clin. Invest. 1994. 94:526-531.) Key words: lipid metabolism • inborn errors $\cdot \beta$-oxidation $\cdot$ genetic code
\end{abstract}

\section{Introduction}

Peroxisomes are subcellular organelles with an important function in cellular metabolic processes including the $\beta$-oxidation of very long chain fatty acids (1) and the synthesis of bile acids (2) and ether-phospholipids (3). The importance of peroxisomes for cellular metabolism is stressed by the discovery of a number of serious inborn errors of peroxisomal metabolism in humans (4-7).

Disorders with defective peroxisome assembly such as the Zellweger cerebro-hepato-renal syndrome (7), neonatal adrenoleukodystrophy (4), and infantile phytanic acid storage disease (5) are characterized by a decreased number or an absence

Address correspondence to Bwee Tien Poll-The, University Children's Hospital, Wilhelmina Kinderziekenhuis, Nieuwegracht 137, 3512 LK Utrecht, The Netherlands. 1994

Received for publication 6 July 1993 and in revised form 1 April

J. Clin. Invest.

(C) The American Society for Clinical Investigation, Inc. 0021-9738/94/08/0526/06 \$2.00

Volume 94, August 1994, 526-531 of morphologically detectable peroxisomes in liver and other tissues. Multiple enzymatic abnormalities, resulting in an accumulation of bile acid intermediates, very long chain fatty acids (VLCFA), ${ }^{1}$ pipecolic, pristanic, and phytanic acids (age-dependent), and severe impairment of plasmalogen biosynthesis, are observed in these disorders (6).

Peroxisomal $\beta$-oxidation of fatty acids is catalyzed by three enzymes that are immunologically distinct from the analogous mitochondrial enzymes: (1) acyl-CoA oxidase; (2) a trifunctional enzyme containing enoyl-CoA hydratase, 3-hydroxy-acyl-CoA dehydrogenase, and enoyl-CoA isomerase activities (previously named bifunctional enzyme; 8 ); and (3) peroxisomal $\beta$-ketothiolase. The peroxisomal $\beta$-oxidation pathway appears to be active toward saturated VLCFA (1). An impairment of this pathway is responsible for the elevated plasma and tissue VLCFA values in patients.

Recently, several patients with defective peroxisomal functions have been described in which peroxisomes were not decreased in number in various tissues. These patients were diagnosed initially with either Zellweger syndrome or neonatal adrenoleukodystrophy based on their clinical and pathological manifestations. However, in contrast to the disorders of peroxisome assembly, these patients appeared to have an isolated defect of the peroxisomal $\beta$-oxidation. One patient with clinical features similar to those of Zellweger patients was found to have an isolated deficiency in peroxisomal thiolase protein. No large DNA rearrangement involving the human peroxisomal thiolase gene was found in this patient (9). Another patient with a single enzyme defect of peroxisomal VLCFA metabolism, namely trifunctional enzyme deficiency, had been diagnosed originally as having neonatal adrenoleukodystrophy (10).

We have described two siblings with pseudoneonatal adrenoleukodystrophy who exhibited decreased VLCFA oxidation associated with an isolated deficiency of fatty acyl-CoA oxidase activity (11), the first enzyme of the peroxisomal $\beta$-oxidation system. We have now initiated an investigation aiming to clone the human acyl-CoA oxidase cDNA and to determine the genetic defect in the two siblings. In this study, we report the sequence of the human acyl-CoA oxidase cDNA and show that the genetic lesion underlying the acyl-CoA oxidase deficiency involves a large DNA deletion in the two patients.

\section{Methods}

Patients. Two previously reported siblings born to consanguineous healthy parents presented clinical manifestations very similar to those

1. Abbreviations used in this paper: SSPE, sodium chloride sodium phosphate EDTA buffer; VLCFA, very long chain fatty acids. 
Table I. Oligonucleotides Used in PCR Amplification

\begin{tabular}{clc}
\hline Primer & \multicolumn{1}{c}{ Sequence 5' to 3' } & Coordinates \\
\hline 1-H13[3] & AAATGCATCAACCAAAGCAAC & $1830-1810$ \\
2-R10[5] & GTCTGGCCAACTATGGTGGAC & $1408-1428$ \\
3-H11[3] & AAGGTTTTTGCAGCAATTTC & $1506-1486$ \\
4-R8[5] & CAGTATAAACTCTTCCCGCTC & $982-1002$ \\
5-H8[3] & TTCGTTAATCCGGTGATAGGT & $1071-1051$ \\
6-R6[5] & ACTGTCGGGGATATCGGTCCC & $664-684$ \\
7-H6[3] & CTGGGCATACTTCATCAGCAT & $774-754$ \\
8-R1[5] & ATGAACCCCGACCTGCGCAAG & $1-21$ \\
9-H1[5] & TCCGCCAGCTTCAACCCGGAG & $31-51$ \\
10-H2[3] & TGCCAAACTCCCTCATCTTC & $235-216$
\end{tabular}

of patients affected by neonatal adrenoleukodystrophy, namely severe hypotonia, mental retardation, seizures, and no dysmorphic features. Standard karyotypes were normal. In contrast to neonatal adrenoleukodystrophy, hepatic peroxisomes were enlarged in size but not decreased in number. $\mathrm{A} \mathrm{C}_{26: 0} / \mathrm{C}_{22: 0}$ ratio of 0.076 and 0.16 was observed in the plasma of each patient as opposed to a ratio of $0.016( \pm 0.028)$ in control plasma, and a ratio of 1.577 and 1.047 in patients' fibroblasts compared with $0.08( \pm 0.03)$ in control fibroblasts. The parents' VLCFA ratios were comparable with those of controls. Immunoblotting experiments on liver tissue from the patients revealed no immunologically reactive material using anti-acyl-CoA oxidase antibodies. The accumulation of VLCFA appeared to be associated with an isolated deficiency of the fatty acyl-CoA oxidase, the enzyme that catalizes the first step of the peroxisomal $\beta$-oxidation. Plasmalogen biosynthesis in cultured skin fibroblasts and plasma levels of di- and trihydroxycoprostanoic acid, phytanic acid, and pipecolic acid were normal (11).

cDNA cloning and sequencing. A $\lambda$-gt10 liver cDNA library $(120,000$ independent recombinant plaques; 12$)$ was screened using PCR-amplified exon 13 of the rat acyl-CoA oxidase gene ( $5^{\prime}$ primer 5'-GGGAGCATCATCACAGGGGCT-3', 3' primer 5'-CTCTGTTTTGTTCAGTGGGGA-3'; 13). The two primary clones selected (insert size of $200 \mathrm{bp}$ ) were subcloned in phage M13mp18 for sequencing by the dideoxy chain termination method of Sanger. A second screening failed to give larger clones. For this reason a different strategy consisting of amplification of reverse-transcribed specific human mRNA was initiated.

Total human liver RNA ( $10 \mu \mathrm{g}$ ) was reverse transcribed to cDNA, using a 21-bp specific primer complementary to the 3 ' end of the human clones obtained previously, using reverse transcriptase (GIBCO BRL, Gaithersburg, MD) as recommended by the manufacturer. For secondstrand cDNA synthesis and PCR amplification of subsequent fragments (Taq-polymerase, $2.5 \mathrm{U}$; Perkin-Elmer Cetus Instruments, Norwalk, CT), forward primers derived from the rat cDNA sequence were selected. Sequence analysis of these fragments provided information for reverse transcription and amplification of the next fragment. This procedure of using specific human oligonucleotides as backward primers and specific rat oligonucleotides as forward primers was repeated. To obtain the 5 ' end of the cDNA, these fragments served as probes for screening a larger human liver cDNA $\lambda$-gt 11 library (14). One single recombinan containing an incomplete insert was selected and used as probe for screening another library (human B cell line cDNA library in pCDM8). A cDNA clone of $2.1 \mathrm{~kb}$ encoding the complete protein was obtained.

Primers used. The synthetic oligonucleotides used in PCR sequencing analysis of the human acyl-CoA oxidase cDNA are shown in Table I. The coordinates (numbering starts at ATG codon) are based on the human $(\mathrm{H})$ (Fig. 1$)$ or the rat $(\mathrm{R} ; 13)$ cDNA sequence.

Southern blotting. Genomic DNA was obtained from lymphoblastoid cell lines by standard methods. Control and patients' genomic DNA $(8 \mu \mathrm{g})$ were digested with either BglII or EcoRI restriction enzymes, electrophoresed in a $0.7 \%$ agarose gel, and transferred to Hybond $\mathrm{N}^{+}$ membranes (Amersham Corp., Arlington Heights, IL) according to the manufacturer's instructions.

Filters were prehybridized for $1 \mathrm{~h}$ and hybridized overnight in a hybridization mix containing $5 \times$ sodium chloride sodium phosphate EDTA buffer (SSPE), $5 \times$ Denhardt's solution, $0.5 \%$ SDS, and 100 $\mu \mathrm{g} / \mathrm{ml}$ herring sperm DNA. Filters were washed in $2 \times \mathrm{SSPE} / 0.1 \%$ SDS, $1 \times \mathrm{SSPE} / 0.1 \% \mathrm{SDS}$, and $0.1 \times \mathrm{SSPE} / 0.1 \% \mathrm{SDS}$ as recommended by the manufacturer.

For hybridizing the Southern blots, the full length cDNA was digested with the restriction enzyme DraI, giving a $5^{\prime}$ fragment (nucleotides 0-264) and a $3^{\prime}$ fragment (nucleotides 265-2092), and both fragments were labeled using the Amersham labeling kit and ${ }^{32} \mathrm{P}$-labeled dCTP. An average of $2.10^{6} \mathrm{cpm} / \mathrm{ml}$ was used per blot. Blots were scanned with a phosphorimager.

\section{Results}

Isolation of the human acyl-CoA oxidase cDNA. To study the molecular defect in our patients with an acyl-CoA oxidase deficiency, we set out to clone a full-length human acyl-CoA oxidase cDNA. To that end, a $\lambda$-gt10 human liver cDNA library was screened with a probe representing exon 13 of the rat acylCoA oxidase gene. The two short human clones ( $\pm 200 \mathrm{bp}$ ) obtained had an $84 \%$ homology to the rat cDNA sequence. Further screening of this library failed to give larger clones. Therefore, we amplified reverse-transcribed human liver mRNA using primers based on the rat cDNA sequence. Most of the cDNA sequence was obtained in three amplification steps using primers 1-H13[3] and 4-R8[5], 3-H11[3] and 6-R6[5], and 7-H6[3] and 8-R1 [5]. This yielded overlapping fragments of 849,843 , and $774 \mathrm{bp}$, respectively. The 5' end, however, remained elusive.

Screening of a $\lambda$-gt 11 human liver cDNA library (containing large inserts) was carried out using PCR fragments as probes and gave an incomplete 1.9-kb insert. Since this insert did not include the $5^{\prime}$ end of the cDNA, a third library (a B cell line cDNA library) was screened. The $2.1-\mathrm{kb}$ insert obtained from this last library encoded the complete protein sequence.

The B cell cDNA sequence contained a region of 160 nucleotides (nucleotides 270-429; Fig. 1), which was different from the cDNA sequence of the $1.9-\mathrm{kb}$ liver cDNA clone. Further PCR amplification using primers 9-H1 [5] and 7-H6[3] on liver mRNA gave a 774-bp fragment with either sequences though never both together, whereas amplification of lymphocyte mRNA gave only one type of sequence. The sequence found only in liver mRNA was called type I, and the sequence found in both liver and lymphocytes mRNA was called type II (Fig. 2).

The nucleotide sequence of the human acyl-CoA oxidase cDNA (Fig. 1) was found to be highly homologous to its rat cDNA counterpart. The homology between the two sequences averaged $85 \%$ at the nucleotide level and $89 \%$ at the amino acid level (Fig. 3). As in the rat (13), two species of acyl-CoA oxidase cDNA were found in the liver differing in the region of nucleotides 270-429 (type I and II; Fig. 2). These two regions had a $52 \%$ homology to each other at the nucleotidic level and each had a $91 \%$ homology to their rat counterpart.

Southern blot analysis of the acyl-CoA oxidase gene in control and patients with an acyl-CoA oxidase deficiency. Southern blot analysis of total human genomic DNA digested with a number of restriction enzymes and using the full-length cDNA as the hybridization probe indicated that the chromosomal acyl-CoA oxidase gene is at least $30-40 \mathrm{~kb}$ long and 
1 atg AAC CCG GAC CTG CGC AGg Gag CGg GAT TCC GCC AGC TTC AAC CCG GAg CTG CTT ACA CAC ATC CTG GAC GGC 1 Met Asn Pro Asp Leu Arg Arg Glu Arg Asp Ser Ala Ser Phe Asn Pro Glu Leu Leu Thr His Ile Leu Asp Gly

76 AGC CTC GAG AAA ACC CGG CGC CGC CGA GAG ATC GAG AAC ATG ATC CTG MAC GAC CCA GAC TTC CAG CAT GAG GAC 26 Ser Leu Glu Lys Thr Arg Arg Arg Arg Glu Ile Glu Asn Met Ile Leu Asn Asp Pro Asp Phe Gln His Glu Asp

151 TTG AAC TTC CTC ACT CGC AGC CAG CGT TAT GAG GTG GCT GTC AGG AAA AGT GCC ATC ATG GTG AAG AAG ATG AGG
51 Leu ABn Phe Leu Thr Arg Ser Gln Arg Tyr Glu Val Ala Val Arg Ly Ser Ala Ile Met Val Lys LyB Met Arg

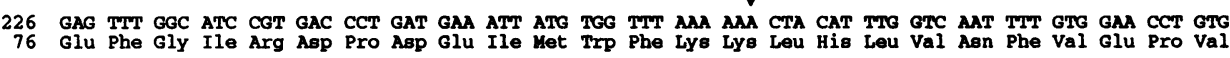
301 CGC CTC AAT TAC TCC ATG TTT ATT CCT ACC TTG CTG AAT CAG GGC ACC ACT GCT CAG AAA GAG AMA TGG CTG CTT 101 Arg Leu Asn Tyr Ser Met phe Ile pro Thr Leu Leu Asn gln Gly Thr Thr Ala gln Lys glu Lys Trp Leu Leu 376 TCA TCC AAA GGA CTC CAG ATA ATT GGC ACC TAC GCC CAG ACG GAA ATG GGC CAC GGA ACT CAC CTT CGA GGC TTG
126 Ser Ser Lys Gly Leu Gln Ile Ile Gly Thr Tyr Ala Gln Thr Glu Met Gly His Gly Thr His Leu Arg Gly Leu 451 GAA ACC ACA GCC ACG TAT GAC CCT GAA ACC CAG GAG TTC ATT CTC AAC AGT CCT ACT GTG ACC TCC ATT AAA TGG 151 Glu Thr Thr Ala Thr Tyr Asp pro glu Thr Gln Glu phe Ile Leu Asn Ser pro Thr Val Thr Ser Ile Lys Trp 526 TGG CCT GGT GGG CTT GGA AAG ACT TCA AAT CAT GCA ATA GTT CTT GCC CAG CTC ATC ACT AAG GGG AAA TGC TAT 176 Trp pro Gly Gly Leu gly Lys Thr Ser Asn His Ala Ile Val Leu Ala Gln Leu Ile Thr Lys Gly Lys Cys Tyr 601 GGA TTA CAT GCC. TTT ATC GTA CCT ATT CGT GAA ATC GGG ACC CAT AAG CCT TTG CCA GGA ATT ACC GTT GGT GAC 676 ATC GGC CCC AAA TTT GGT TAT GAT GAG ATA GAC AAT 3GC TAC CTC AAA ATG GAC AAC CAT CGT ATT CCC AGA GAM 676 ATC GGC CCC AAA TTT GGT TAT GAT GAG ATA GAC AAT 3GC TAC CTC AAA ATG GAC AAC CAT CGT ATT CCC AGA GA
226 Ile Gly Pro Lys phe Gly Tyr Asp Glu Ile Asp Asn Gly Tyr Leu Lys Met Asp Asn His Arg Ile Pro Arg Glu

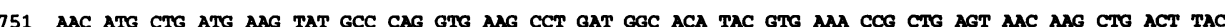
251 Asn Met Leu Met Lys Tyr Ala Gln Val Lys Pro Asp Gly Thr Tyr Val Lys Pro Leu Ser Asn Lys Leu Thr Tyr 826 GGG ACC ATG GTG TTT GTC AOG TCC TTC CTT GTG GGA GAA GCT GCT CGG GCT CTG TCT AAG GCG TGC ACC ATT GCC 276 Gly Thr Net Val Phe Val Arg Sor The Lou Val Gly Glu Ala Mla Arg Ala Leu Ser Lys Ala Cys Thr Ilo Ala

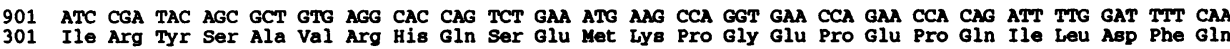
976 ACC CAG CAg TAT AAA CTC TTT CCA CTC CTG GCC ACT GCC TAT GCC TTC CAG TTT GTG GGC GCA TAC ATG Aag GAG 326 Thr Gin Gln Tyr Lys Leu Phe Pro Leu Leu Ala Thr Ala Tyr Ala phe Gln Phe Val Gly Ala Tyr Met Lys Glu 1051 ACC TAT CAC CGG ATT AAC GAA GGC ATT GGT CAA GGG GAC CTG AGT GAA CTG CCT GAG CTT CAT GCC CTC ACC GCT 351 Thr Tyr his Arg Ile Asn Glu Gly Ile Gly Gln Gly Asp Leu Ser Glu Leu Pro glu Leu His Ala Leu Thr Ala 1126 GGA CTG AAG GCT TPC ACC TCC TGG ACT GCA AAC ACT GGC ATT GAA GCA TGT CG ATG GCT TGT GGT GGE CAT GGC 201 TAT TCT CAT TGC AGT GGT CTT CCA AAT ATT TAT GTC AAT TTC ACC CCA AGC TGT ACC TPT GAG GGA GAA AAC ACT 401 Tyr Ser his Cys Ser gly Leu Pro Aan Ile tyr Val Aan phe Thr pro Ser Cys Thr phe Glu Gly Glu Asn Thr 1276 GTC ATG ATG CTC CAG ACG GCT AGG TTC CTG ATG AMA AGT TAT GAT CAG GTG CAC TCA GGA AAG TTG GTG TGT GGC 426 Val Met Met Leu Gln Thr Ala Arg phe Leu Het Lys Ser Tyr Asp gln Val his Ser gly Lys Leu Val Cys Gly 1351 ATG GTG TCC TAT TTG AAC GAC CTG CCC AGT CAG CGC ATC CAG CCA CAG CAG GTA GCA GTC TGG CCA ACC ATG GTG 1426 GAT ATC AAC AGC CCC GAA AGC CTA ACC GAA GCA TAT AMA CTC COT GCA GCC AGA TTA GTA GAA ATT GCT GCA AA 1426 GAT ATC AAC AGC CCC GAA AGC CTA ACC GAA GCA TAT AAA CTC COT GCA GCC AGA TPA GTA GAA ATT GCT GCA AAA
476 Asp Ile Asn Ser Pro Glu Ser Leu Thr Glu Ala Tyr Lyb Leu Arg Ala Ala Arg Leu Val Glu Ile Ala Ala Lys 1501 AAC CTT CAA AAA GAA GTG ATT CAC AGA AAA AGC AAG GAG GTA GCT TGG AAC CTA ACT TCT GTT GAC CTT GTT CGA 501 Asn Leu Gln Lys Glu Val Ile His Arg Lys Ser Lys Glu Val Ala Trp Asn Leu Thr Ser Val Asp Leu Val Arg 1576 GCA AGT GAG GCA CAT TGC CAC TAT GTG GTA GTT AAG CTC TTT TCA GAM AAA CTC CTC AAA ATT CAA GAT AAA GCC 526 Ala Ser Glu Ala his Cys his Tyr Val Val Val Lys Leu phe Ser Glu Lys Leu Leu Lys Ile Gln Asp Lys Ala 1651 ATT CAA GCT GTC TTA AGG AGT TTA TGT CTG CTG TAT TCT CTG TAT GGA ATC AGT CAG AAC GCG GGG GAT TTC CTT 1726 CAG GGg AGC ATC ATG ACA GAg CCT CAg ATT ACA CAN GTA AAC CAG CGT GTA AAG GA TTA CTC ACT CTG ATT COC

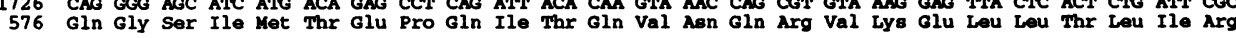
1801 TCA GAT GCT GTT GCT TFG GTT GAT GCA TTP GAT TTT CAG GAT GCB ACA CTT GGC TCT GTO CTP GOC CGC TAT GAT 601 Ser Asp Ala Val Ala Leu Val Asp Ala phe Asp phe Gln Asp Ala Thr Leu Gly Ser Val Leu Gly Arg Tyr Asp 1876 GGG AAT GTG TAT GAA AAC TTG TTT GA TGG GCT AAG AAC TCC CCA CTG AAC AAA GCA GAG GTC CAC GAA TCT TAC 626 Gly Asn Val Tyr Glu Asn Leu phe Glu Trp Ala Lys Asn Ser Pro Leu Asn Lys Ala glu Val His Glu Ser Tyr

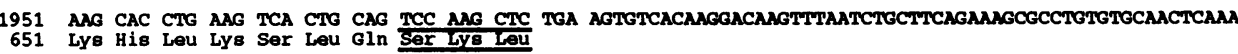

2076 THTTGTGGATCTTTTT

Figure 1. Nucleotide and predicted amino acid sequences of the cDNA encoding the human acylCoA oxidase. Numbering of nucleotides begins at the ATG codon. Only type I sequence is shown (see Fig. 2). The underlined three amino acids correspond to the peroxisomal targeting signal found in many species. Filled triangles denote the beginning and the end of the type I specific sequence. The sequence has been submitted to the EMBL gene bank (Heidelberg); accession no. X71440. therefore contained multiple intervening sequences. The occurrence of RFLP of the human acyl-CoA oxidase gene was investigated by Southern blotting of genomic DNA from 23 families digested with restriction enzymes BamHI, BglII, EcoRI,
EcoRV, HindIII, MspI, PstI, PvuII, RsaI, TaqI, and XmnI and hybridization with the cDNA. None of these enzymes detected any RFLP, which would have enabled linkage studies.

Total DNA, derived from either cultured skin fibroblasts or

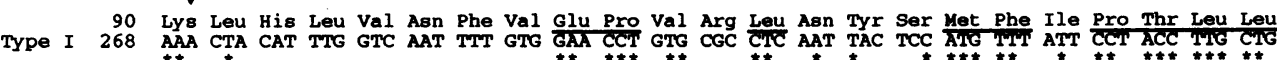

Type II 268 AAT TPT GTG CAC CGA GGG CGg CCT GAG CCT CTG GAT CTT CAC TTG GGC ATG TTC CTG CCC ACC TTG CTT

90 Asn phe Val His Arg Gly Arg Pro Glu Pro Leu Asp Leu His Leu Gly Met Phe Leu Pro Thr Leu Leu

Type I 337 AAT CAG GGC Thr Thr Ala Gln LyB Glu LYs Trp Leu Leu Ser Ser Lys Gly Leu Gln Ile Ile Gly Thr

Type II 337 CAC CAG

113 His Gln Ala Thr Ala Glu Gln Gln Glu Arg Phe Phe Met Pro Ala Trp Asn Leu Glu Ile Ile Gly Thr

136 Tyr Ala Gln Thr Glu Met Gly His Gly

Type I 406 THC GCC CAG TCG GAN AIG GGC CAC GGA

Type II 406 TAT GCC CAG ACA GAG ATG GGT CAT GGA

136 Tyr Ala Gln Thr Glu Net Gly His Gly
}

Figure 2. Type I and type II specific cDNA sequences and corresponding amino acid sequences. The numbering is identical to Fig. 1. Filled triangles delineate the borders of the differences between the two sequences. Matching nucleotides between the two sequences are marked with asterisks, and matching amino acids are underlined. 


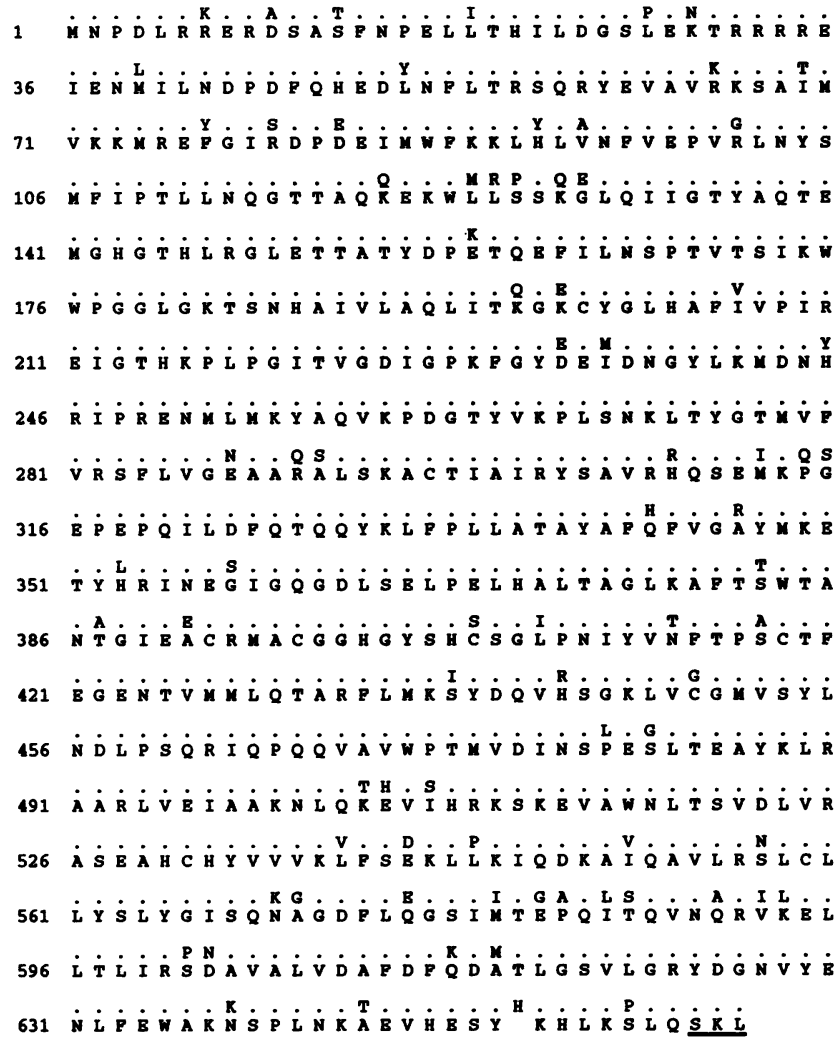

Figure 3. Comparison between the predicted amino acid sequences of the human and the rat acyl-CoA oxidase type I. The human amino acid sequence is given in single letter code. Amino acid identity between the rat and human sequence is indicated by a dot above the human sequence and mismatches by the corresponding rat amino acid. The numbering indicated is that of the human amino acid sequence.

lymphoblastoid cell lines from the siblings with an acyl-CoA oxidase deficiency, their parents, and controls, was digested with restriction enzymes BgIII, EcoRI, and HindIII and probed with the cDNA. A partial deletion of the acyl-CoA oxidase gene was detected in the patients, regardless of the restriction enzyme used. Patients' DNA digested with restriction enzyme EcoRI lacked the 8.0-, 5.5-, and 2.4-kb fragments when probed with the 3 ' fragment including the type II region (nucleotides 2652092; Fig. $4 A$ ). The same blot was reprobed with a 5 ' fragment from the human clone, homologous to the first two exons of the rat gene (nucleotides 0-264). A 16-kb fragment was now found to be present in patients as well as in controls (Fig. 4 $B$ ). This observation was confirmed by PCR amplification using oligonucleotides 9-H1[5] and 11-H2[3], both situated at the 5 ' end of the cDNA, on genomic DNA from patients and controls. A 500-bp fragment was found to be present both in the probands and in the controls. This fragment was sequenced and apparently included an intron at a position equivalent to the rat intron 1.

Although the exact boundaries of the deletion remain to be determined, we conclude that the deletion in the patients spans most of the gene as observed by Southern blot analysis. However, the first two exons were left intact. These results indicate that the acyl-CoA oxidase deficiency in this family is due to a large deletion in the acyl-CoA oxidase gene.

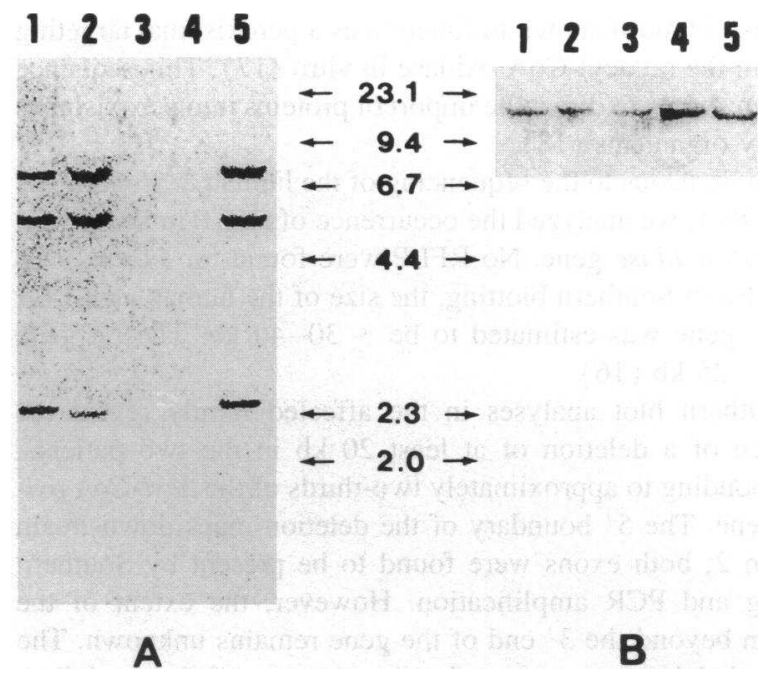

Figure 4. Southern blot analysis of the affected family. DNA from control (lane 1), the mother (lane 2), the two patients (lanes 3 and 4), and a Zellweger patient (lane 5) was digested with the restriction enzyme EcoRI and probed with a 1827-bp cDNA fragment (nucleotides 265-2092) ( $A$ ) or a 264-bp cDNA fragment (nucleotides 0-264) which covers exons 1 and $2(B) .8 .0-, 5.5-$, and 2.4-kb fragments were found to be missing in patients' DNA $(A)$, whereas a 16-kb fragment was present $(B)$. The pattern observed for the Zellweger patient DNA was identical to control DNA. $\lambda$-DNA digested with HindIII is shown as a marker. Inspection of the ethidium bromide-stained gel revealed that comparable amounts of DNA were loaded in each lane (not shown). Similar results were obtained with a BglII digest (not shown).

\section{Discussion}

The human acyl-CoA oxidase gene has not been characterized previously. However, the rat acyl-CoA oxidase gene (13) and the yeast Candida tropicalis POX 4 and POX 5 genes (15) have been characterized. Significant similarities between the amino acid sequences of the rat acyl-CoA oxidase and the yeast POX 4 and POX 5 enzymes are reported. Particularly, the $\mathrm{COOH}$-terminal end of these enzymes (amino acid position 622-634 in the rat enzyme) displayed a high degree of homology. Based on these observations, exon 13 of the rat acyl-CoA oxidase was amplified and used as a probe to screen human cDNA libraries. Subsequent PCR cloning and cDNA library screens eventually allowed us to isolate a full-length acyl-CoA oxidase cDNA.

Sequence analysis of the human acyl-CoA oxidase cDNA revealed an $85 \%$ homology with the rat nucleotidic sequence. Interestingly, we have observed that one codon of the rat exon 14 (nucleotides 1951-1953) is absent in the human cDNA sequence. We have also observed the presence of two cDNAs in the liver differing in the region of nucleotides 270-429, a region which corresponds to an alternative usage of rat exon 3 (16). The presence of both types of mRNA in the liver but not in lymphocytes may be related to the essential role of liver peroxisomes in $\beta$-oxidation of VLCFA.

An $89 \%$ homology was found between the deduced amino acid sequences of rat and human acyl-CoA oxidase genes. The carboxy termini of the human and rat acyl-CoA oxidase enzyme are highly homologous $(88 \%$ homology in the last 50 amino acids). Like the rat homologue, the human sequence also contains the carboxy-terminal Ser-Lys-Leu (SKL) (residues 656- 
660, Fig. 1) motif shown to function as a peroxisomal targeting signal in the rat acyl-CoA oxidase in vitro (17). This sequence has been shown to direct the import of proteins into peroxisomes in many organisms (18).

Simultaneous to the sequencing of the human acyl-CoA oxidase cDNA, we analyzed the occurrence of RFLP in the human acyl-CoA oxidase gene. No RFLP were found for 11 enzymes tested. From Southern blotting, the size of the human acyl-CoA oxidase gene was estimated to be $\sim 30-40 \mathrm{~kb}$. The rat gene spans $\sim 25 \mathrm{~kb}(16)$.

Southern blot analyses in the affected family reveal the presence of a deletion of at least $20 \mathrm{~kb}$ in the two patients, corresponding to approximately two-thirds of the acyl-CoA oxidase gene. The $5^{\prime}$ boundary of the deletion maps downstream of exon 2; both exons were found to be present by Southern blotting and PCR amplification. However, the extent of the deletion beyond the $3^{\prime}$ end of the gene remains unknown. The observed deletion accounts for the absence of the acyl-CoA oxidase protein shown by immunoblotting experiments on liver tissues from one of the patients (11). The detection of $25 \%$ residual enzymatic activity in patient's liver, however, is likely due to the activity of trihydroxycoprostanoyl-CoA acid oxidase (19). The normal plasma values of di- and trihydroxycoprostanoic acids are indirect evidence of the normal activity of the trihydroxycoprostanoyl-CoA oxidase enzyme in the patients.

Very little is known about the molecular basis of peroxisomal diseases, as peroxisome research is a relatively new field. The precise cause of the defects remains unknown in many cases of generalized peroxisomal deficiencies. However, the specific genes involved and the molecular defects are being elucidated gradually and, as expected, include both DNA rearrangements and point mutations.

Disorders with multiple peroxisomal dysfunction (Zellweger syndrome, neonatal adrenoleukodystrophy, and infantile Refsum disease) can be classified in at least eight complementation groups (20), suggesting a multigenic origin of the disease. A microdeletion of the proximal long arm (21) and a pericentric inversion of chromosome 7 (22) have been observed in two unrelated patients affected by Zellweger syndrome, a disorder of peroxisome assembly. These findings have led to the tentative assignment of one of the genes responsible for Zellweger syndrome to $7 \mathrm{q} 11.23$.

Recently, point mutations were found in two peroxisomal membrane proteins in Zellweger syndrome patients. One patient had a point mutation in the $35-\mathrm{kD}$ membrane protein-coding sequence (23). Two other patients had a splice site or missense mutation in the gene of the $70-\mathrm{kD}$ membrane protein (24).

A patient suffering from a single enzyme defect in the peroxisomal $\beta$-oxidation did not display a detectable DNA rearrangement of the human peroxisomal $\beta$-ketothiolase gene in genomic DNA (9). This patient had more serious clinical symptoms than our patients which may be due to the peroxisomal thiolase involvement in the $\beta$-oxidation of di- and trihydroxycoprostanoic acids as well as of VLCFA.

Recently, a rearrangement altering the color pigment genes in a patient with X-linked adrenoleukodystrophy was shown to include two deletions apparently separated by a large inversion on chromosome $\mathrm{Xq} 28$ (25). Further investigation of this region led to the cloning of a gene with significant homology to a peroxisomal 70-kD membrane protein which was partially deleted in $7 \%$ of $85 \mathrm{X}$-linked adrenoleukodystrophy patients (26). $\mathrm{X}$-linked adrenoleukodystrophy is now thought to be associated with a defect in the transport of the peroxisomal acyl-CoA synthetase, the enzyme responsible for the activation of VLCFA before their $\beta$-oxidation in the peroxisome (27).

The present study reports the existence of a large deletion in two related patients with a deficiency in the peroxisomal acyl-CoA oxidase. This is the first molecular defect found to be associated with this disease. Additional patients, unrelated to the probands of this study, have been identified by using complementation analysis between cell lines of unknown patients with those of patients with known specific enzymatic defects in the VLCFA $\beta$-oxidation (28). The available human acyl-CoA oxidase cDNA sequence will allow the determination of additional molecular defects resulting in this disease.

\section{Acknowledgments}

We thank W. de Lau (Department of Immunology, University Hospital Utrecht) for providing us with a human B cell cDNA library.

Part of this work was funded by the Association Française contre les Myopathies.

\section{References}

1. Singh, I., A. E. Moser, S. Goldfischer, and H. W. Moser. 1984. Lignoceric acid is oxidized in the peroxisome: implications for the Zellweger cerebro-hepatorenal syndrome and adrenoleukodystrophy. Proc. Natl. Acad. Sci. USA. 81:42034207.

2. Björkhem, I., B. F. Kase, and J. I. Pedersen. 1985. Role of peroxisomes in the biosynthesis of bile acids. Scand. J. Clin. Lab. Invest. 45(Suppl. 177):2331 .

3. Hajra, A. K., and J. E. Bishop. 1982. Glycerolipid biosynthesis in peroxisomes via the acyl dihydroxyacetone phosphate pathway. Ann. NY Acad. Sci. 386:170-182.

4. Kelley, R. I., N. S. Datta, W. B. Dobyns, A. K. Hajra, A. B. Moser, M. J. Noetzel, E. H. Zackai, and H. W. Moser. 1986. Neonatal adrenoleukodystrophy: new cases, biochemical studies, and differentiation from Zellweger and related peroxisomal polydystrophy syndromes. Am. J. Med. Genet. 23:869-901.

5. Poll-The, B. T., J. M. Saudubray, H. A. M. Ogier, M. Odièvre, J. M. Scotto, L. Monnens, L. C. P. Govaerts, F. Roels, A. Cornelis, R. B. H. Schutgens, et al. 1987. Infantile Refsum disease: an inherited peroxisomal disorder. Comparison with Zellweger syndrome and neonatal adrenoleukodystrophy. Eur. J. Pediatr. 146:477-483.

6. Wanders, R. J. A., H. S. A. Heymans, R. B. H. Schutgens, P. G. Barth, H. van den Bosch, and J. M. Tager. 1988. Peroxisomal disorders in neurology. $J$. Neurol. Sci. 88:1-39.

7. Goldfisher, S., C. L. Moore, A. B. Johnson, A. J. Spiro, M. P. Valsamis, H. K. Wisniewski, R. H. Ritch, W. T. Norton, I. Rapin, and L. M. Gartner. 1973. Peroxisomal and mitochondrial defects in the cerebro-hepato-renal syndrome. Science (Wash. DC). 182:62-64.

8. Palosaari, P. M., and J. K. Hiltunen. 1990. Peroxisomal bifunctional protein from rat liver is a trifunctional enzyme possessing 2-enoyl-CoA hydratase, 3hydroxyacyl-CoA dehydrogenase, and delta 3, delta 2-enoyl-CoA isomerase activities. J. Biol. Chem. 265:2446-2449.

9. Bout, A., M. M. Franse, J. Collins, L. Blonden, J. M. Tager, and R. Benne. 1991. Characterization of the gene encoding human peroxisomal 3-oxoacyl-CoA thiolase (ACAA). No large DNA rearrangement in a thiolase-deficient patient. Biochim. Biophys. Acta. 1090:43-51.

10. Watkins, P. A., W. W. Chen, C. J. Harris, G. Hoefler, S. Hoefler, D. C. Blake, Jr., A. Balfe, R. I. Kelley, A. B. Moser, M. E. Beard, and H. W. Moser. 1989. Peroxisomal bifunctional enzyme deficiency. J. Clin. Invest. 83:771-777.

11. Poll-The, B. T., F. Roels, H. Ogier, J. Scotto, J. Vamecq, R. B. H. Schutgens, R. J. A. Wanders, C. W. T. van Roermund, M. J. A. van Wijland, A. W. Schram, et al. 1988. A new peroxisomal disorder with enlarged peroxisomes and a specific deficiency of acyl-CoA oxidase (pseudo-neonatal adrenoleukodystrophy). Am. J. Hum. Genet. 42:422-434.

12. Lyonnet, S., C. Caillaud, F. Rey, M. Berthelon, J. Frezal, J. Rey, and A. Munnich. 1989. Molecular genetics of phenylketonuria in Mediterranean countries: a mutation associated with partial phenylalanine hydroxylase deficiency. Am. J. Hum. Genet. 44:511-517.

13. Miyazawa, S., H. Hayashi, M. Hijikata, N. Ishii, S. Furuta, H. Kagamiyama, T. Osumi, and T. Hashimoto. 1987. Complete nucleotide sequence of the cDNA and predicted amino acid sequence of the rat acyl-CoA oxidase. J. Biol. Chem. 262:8131-8137. 
14. Le Bouc, Y., D. Dreyer, F. Jaeger, M. Binoux, and P. Sondermeyer. 1986. Complete characterization of the human IGF-1 nucleotide sequence isolated from a newly constructed adult liver cDNA library. FEBS (Fed. Eur. Biochem. Soc.) Lett. 196:108-112.

15. Okazaki, K., T. Tahechi, N. Kambara, S. Fukui, I. Kubota, and T. Kamiryo. 1986. Two acyl-coenzyme A oxidases in peroxisomes of the yeast Candida tropicalis: primary structures deduced from genomic DNA sequence. Proc. Natl. Acad. Sci. USA. 83:1232-1236.

16. Osumi, T., N. Ishii, S. Miyazawa, and T. Hashimoto. 1987. Isolation and structural characterization of the rat acyl-CoA oxidase gene. J. Biol. Chem. 262:8138-8143.

17. Miura, S., I. Kasuya-Arai, H. Mori, S. Miyazawa, T. Osumi, T. Hashimoto and Y. Fujiki. 1992. Carboxyl-terminal consensus Ser-Lys-Leu-related tripeptide of peroxisomal proteins functions in vitro as a minimal peroxisome-targeting signal. J. Biol. Chem. 267:14405-14411.

18. Gould, S. J., G. A. Keller, M. Schneider, S. H. Howell, L. J. Garrard, J. M. Goodman, B. Distel, H. Tabak, and S. Subramani. 1990. Peroxisomal protein import is conserved between yeast, plants, insects and mammals. EMBO (Eur. Mol. Biol. Organ.) J. 9:85-90.

19. Casteels, M., L. Schepers, P. P. van Veldhoven, H. J. Eyssen, and G. P. Mannaerts. 1990. Separate peroxisomal oxidases for fatty acyl-CoAs and trihydroxycoprostanoyl-CoA in human liver. J. Lipid Res. 31:1865-1872.

20. Shimozawa, N., Y. Suzuki, T. Orii, A. Moser, H. Moser, and R. J. A. Wanders. 1993. Standardization of complementation grouping of peroxisomedeficient disorders and the second Zellweger patient with Peroxisomal Assembly Factor (PAF-1) defect. Am. J. Hum. Genet. 52:843-844.

21. Naritomi, K., N. Hyakuna, Y. Suzuki, T. Orii, and K. Hirayama. 1988.
Zellweger syndrome and a microdeletion of the proximal long arm of chromosome 7. Hum. Genet. 80:201-202.

22. Naritomi, K., Y. Izumikawa, S. Ohshiro, K. Yoshida, N. Shimozawa, Y. Suzuki, T. Orii, and K. Hirayawa. 1989. Gene assignment of Zellweger syndrome to 7q11.23: report of the second case associated with a pericentric inversion of chromosome 7. Hum. Genet. 84:79-80.

23. Shimozawa, N., Y. Suzuki, T. Orii, Y. Shirayoshi, T. Mori, and Y. Fujiki. 1992. A human gene responsible for Zellweger syndrome that affects peroxisome assembly. Science (Wash. DC). 255:1132-1134.

24. Gärtner, J., H. Moser, and D. Valle. 1992. Mutations in the $70 \mathrm{kD}$ peroxisomal membrane protein gene in Zellweger syndrome. Nature Genet. 1:16-23.

25. Feil, R., P. Aubourg, J. Mosser, A. M. Douar, D. Le Paslier, C. Philippe, and J. L. Mandel. 1991. Adrenoleukodystrophy: a complex chromosomal rearrangement in the Xq28 red/green-color-pigment gene region indicates two possible gene localizations. Am. J. Hum. Genet. 49:1361-1371.

26. Aubourg, P., J. Mosser, A. M. Douar, C. O. Sarde, J. Lopez, and J. L. Mandel. 1993. Adrenoleukodystrophy gene. Unexpected homology to a protein involved in peroxisome biogenesis. Biochimie (Paris). 75:293-302.

27. Mosser, J., A. M. Douar, C. O. Sarde, P. Kioschis, R. Feil, H. Mosser, A. M. Poustka, J. L. Mandel, and P. Aubourg. 1993. Putative X-linked adrenoleukodystrophy gene shares unexpected homology with $\mathrm{ABC}$ transporters. Nature (Lond.). 361:726-730.

28. Suzuki, Y., N. Shimozawa, S. Yajima, S. Tomatsu, N. Kondo, Y. Nakada S. Akaboshi, M. Iai, Y. Tanabe, T. Hashimoto, et al. 1994. Novel subtype of peroxisomal acyl-CoA oxidase deficiency and bifunctional enzyme deficiency with detectable enzyme protein: identification by means of complementation analysis. Am. J. Hum. Genet. 54:36-43. 